\title{
Anatomy of thoracoscopic thymectomy; case report
}

Anuradha B Jayathilake ${ }^{1}$, UDP Madushanka ${ }^{1}$, V Pinto1, KB Galketiya ${ }^{1}$

${ }^{\text {I} T e a c h i n g ~ H o s p i t a l ~ P e r a d e n i y a, ~ S r i ~ L a n k a ~}$

\begin{abstract}
Thymectomy is performed for myasthenia gravis with or without thymoma. Performing by thoracoscopy reduces the surgical morbidity. Anatomical landmarks play a key role to perform a safe surgery. We present the anatomical highlights of a patient who underwent the procedure
\end{abstract}

\section{Introduction}

Thymectomy improves mysthenia gravis(MG) and may induce remission or reduce the doses of pharmacological treatment(1). Traditional ways of doing Thymectomy are trans-sternal or transcervical. How ever if done by thoracoscopy, morbidity of open surgery will be reducced(2).

We discuss a patient who underwent Thoracoscopic Thymectomy for MG with a thymoma who was also dependent on steroids.

\section{Case report}

The patient being discussed is a 55 year old female diagnosed with MG. On cross sectional imaging he was found to have a thymoma. She required several ICU admisions to control myesthenic crisis. Further she was also dependent on steroids. (Figure 1)

\section{Procedure}

\section{Patient positioning}

After general anasthesia with endotracheal intubation patient was placed on supine position with abduction of right upper limb over the head to expose the axilla.

\section{Ports placement}

3 ports were inserted using following land marks in an inverted triangle in third, fifth and seventh intercostal spaces. (Figure 2)

\section{Creating space for dissection}

To obtain space for dissection, pneumothorax $8 \mathrm{mmHg}$ was induced by insuflation of carbon dioxide.

\section{Identifying and demostration of anatomical land marks}

Following anatomical structures were identified; right brachiochephalic vein, it's union with left brachiochephalic vein, superior vena cava, phrenic nerve and internal mamary vessels which are running downwards over the right side of the pericardium. The magnified image allows clear identification of anatomy. Anatomical relations of above are as follows. (Figure 3, Figure 4) 


\section{Surgical dissection}

After demostrating the anatomy clearly, dissection was started with incision of parital pleura over the superior and anterior mediastinum by using coagulation diathermy. Then proceeded with dissecting around the thymus with surrounding fatty tissue by using bipolar diathermy and ultrasonic dissector. Thymic vein was clipped and divided. Specimen was retrieved using a retrieval bag. $22 \mathrm{G}$ intercostal tube was inserted through one of $5 \mathrm{~mm}$ port and kept it for 1 day.

\section{Post operative}

Patient was managed on the first day in the intensive care unit on spontaneous breathing. She had minimal analgesic requirements, oral feeding commenced on same day. She was discharged on the fifth post operative day.

\section{Discussion}

Traditional ways of doing Thymectomy are trans-sternal and trans-cervical approaches associated with a higher morbidity. In contrast in thoracoscopic approach, there is minimal incision, minimal pain, less wound related complications and early retern to normal activities. This was seen our patient.

In thoracoscopic thymectomy usually the access to the thymus(thymoma) is through right hemithorax. But in some cases where thymoma is more towards left side approach is through the left hemithorax. Here we approached through right hemithorax.
Standard way of getting space for Thoracotomy or Thoracoscopic procedure is single lung ventilation with the use of a double lumen endotracheal intubation and complete collapse of ipsilateral lung. However this needs more expertise and has some associated complications. The technique we use is with a single lumen tube ventilating both lungs and obtaining a partial lung collapse using a capnothorax. This is easy to perform, less complicated while providing adequate space for dissection.(3)

Clear identification and demostration of anatomical structures is necessary prior to initiation of dissection. Those structures are right brachiochephalic vein, it's union with left brachiochephalic vein, superior vena cava, phrenic nerve and internal mammary vessels which are running downwards over the right side of the pericardium. With thoracoscopy perception of Anatomy is easy due to magnified image and ability to zoom closer as demonstrated in this patient.

\section{Conclusion}

The patient presented illustrates that clear identification of anatomical land marks and a safe dissection is possible during thoracoscopic thymectomy and is associated with a lower morbidity.

\section{Prof KB Galketiya, Professorial unit, Teaching hospital, Peradeniya.}

kbgalketiya@yahoo.com

Submitted by - May 2019

Accepted by - July 2019 


\section{References}

1. Wolfe GI, Kaminski HJ, Aban IB, Minisman G, Kuo HC, et al. Randomized Trial of Thymectomy in Myasthenia Gravis. N Engl J Med. 2016Aug 11.375(6):511-22.

2. Lei Yu,Xiao-jun Zhang,Shan Ma,Fei Li,Yun-feng Zhang. Thoracoscopic Thymectomy for
Myasthenia Gravis With and Without Thymoma: A Single-Center Experience. The Anals of Thorasic Surgery. 2011

3. V.Pinto, KB Galketiya,Insufflation pressure required for thoracoscopic surgery and its influence on respiratory and cardiovascular parameters.Sri Lankan Journal of Anaesthesiology 2014;22(2):55-58

\section{Figures}

Figure 1

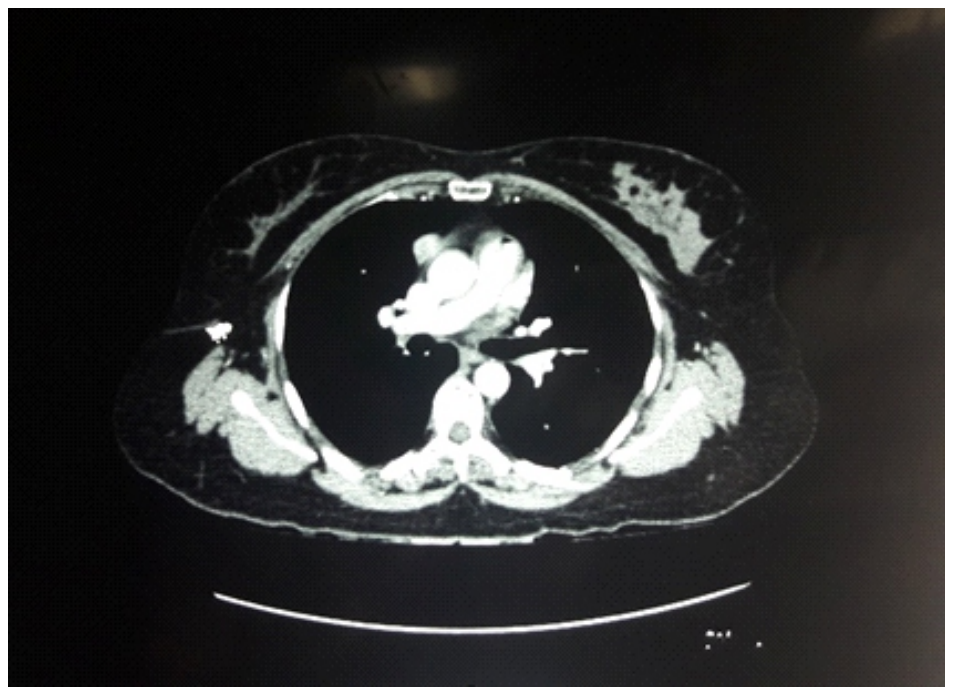

Figure 2

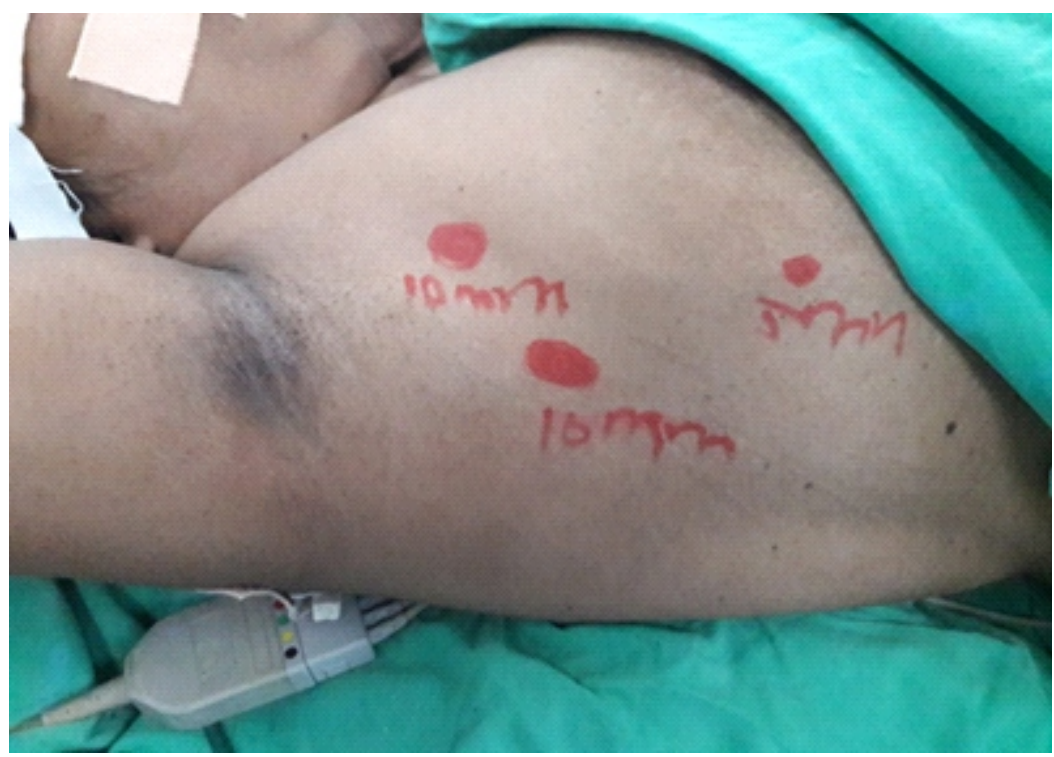


Figure 3

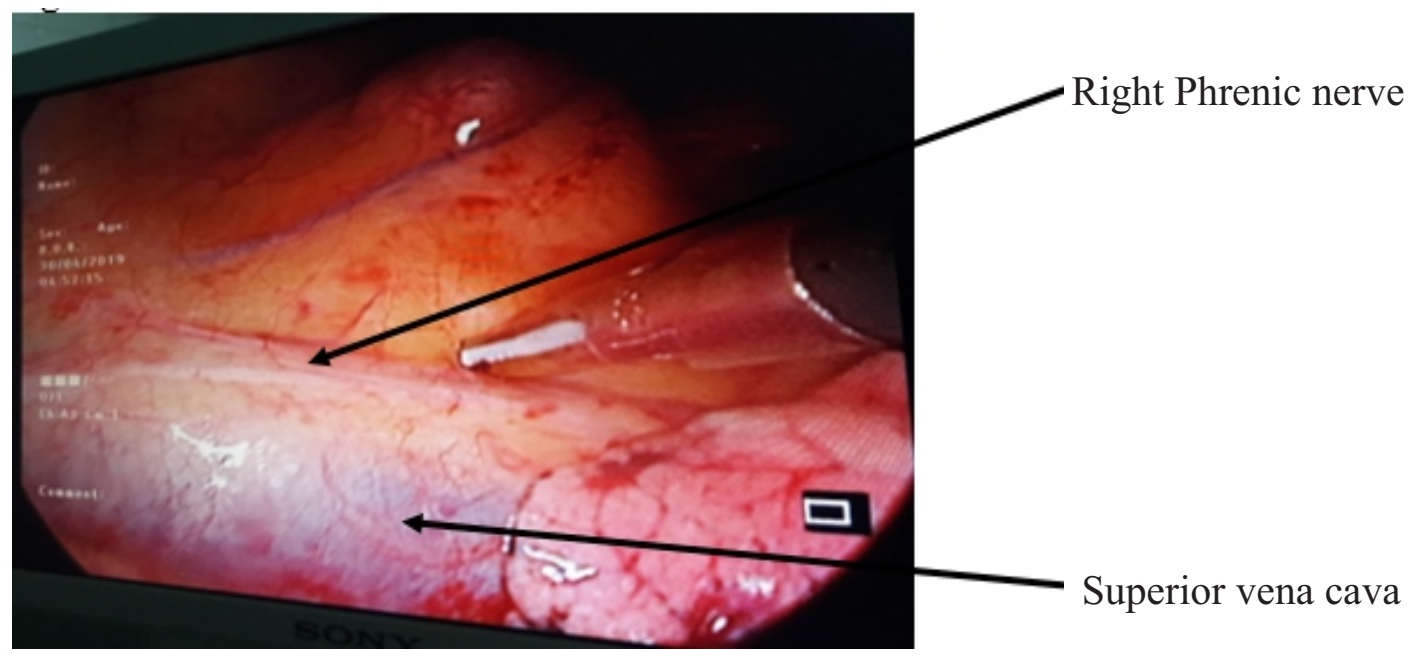

Figure 4

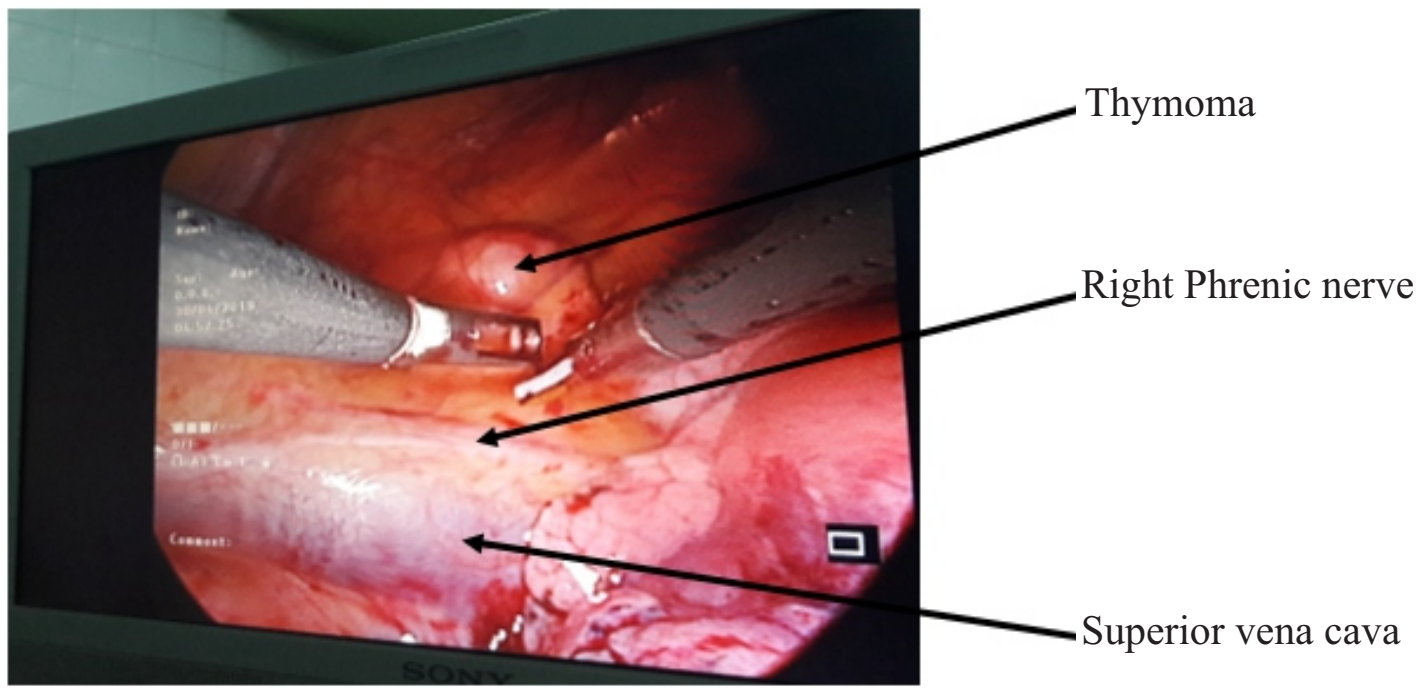

